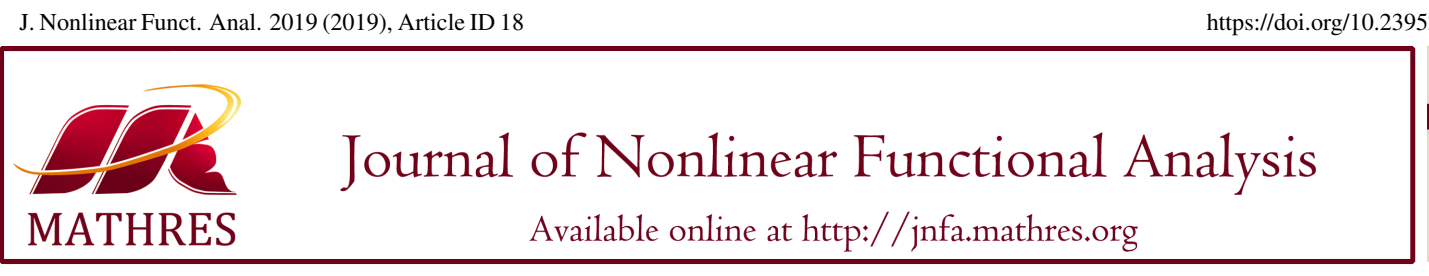

\title{
PERIODIC SOLUTIONS OF IMPULSIVE DIFFERENTIAL EQUATIONS WITH INFINITE DELAY IN BANACH SPACES
}

\author{
JIN LIANG ${ }^{1, *}$ JAMES H. LIU² ${ }^{2}$ MINH VAN NGUYEN ${ }^{3}$, TI-JUN XIAO ${ }^{4}$ \\ ${ }^{1}$ School of Mathematical Sciences, Shanghai Jiao Tong University, Shanghai 200240, China \\ ${ }^{2}$ Department of Mathematics, James Madison University, Harrisonburg, VA 22807, USA \\ ${ }^{3}$ Department of Mathematics and Statistics, University of Arkansas at Little Rock, Little Rock, AR 72204, USA \\ ${ }^{4}$ Shanghai Key Laboratory for Contemporary Applied Mathematics, \\ School of Mathematical Sciences, Fudan University, Shanghai 200433, China
}

\begin{abstract}
In this paper, we investigate the existence of a periodic solution to the Cauchy problem for a class of impulsive differential equations with infinite delay in Banach spaces under general fading memory phase spaces satisfying a basic axiom. It is shown that the related Poincaré operator given by $\mathbb{P}(\phi)=u_{T}(\phi)$ (i.e., $T$ units along the unique solution $u(\phi)$ determined by the initial function $\phi$ ) is a condensing operator by virtue of the Kuratowski measure of non-compactness. Based on this result, we derive the existence of a periodic solution to the Cauchy problem with help of the boundedness of the solutions and three-set fixed point theorems. The main result in this paper extends the previous results for equations without impulsive conditions or in special $C_{g}$ phase spaces. Finally, we give a remark on future developments of the related impulsive problems for fractional differential equations with infinite delay in Banach spaces.
\end{abstract}

Keywords. Monotone operator; Fading memory phase space; Bounded and periodic solution; Impulsive; Fractional differential equation.

2010 Mathematics Subject Classification. 34K30, 34G20, 37C25, 47H08.

\section{INTRODUCTION}

In this paper, we investigate the existence of $T$-periodic solutions to the following Cauchy problem for an impulsive differential equation with infinite delay in a Banach space $(X,\|\cdot\|)$,

$$
\begin{aligned}
& u^{\prime}(t)+A(t) u(t)=f\left(t, u(t), u_{t}\right), t>0, t \neq t_{i}, \\
& u(s)=\phi(s), \quad s \leq 0, \\
& \Delta u\left(t_{i}\right)=I_{i}\left(u\left(t_{i}\right)\right), \quad i=1,2, \cdots, 0<t_{1}<t_{2}<\cdots<+\infty,
\end{aligned}
$$

${ }^{*}$ Corresponding author.

E-mail address: jinliang@sjtu.edu.cn (J. Liang).

Received December 24, 2018; Accepted April 26, 2019. 
where $A(t)$ and $f(t, x, y)$ are $T$-periodic in $t$, the operator $A(t)$ is unbounded,

$$
\begin{gathered}
u_{t}(s)=u(t+s), \quad s \leq 0, \\
\Delta u\left(t_{i}\right)=u\left(t_{i}^{+}\right)-u\left(t_{i}^{-}\right),
\end{gathered}
$$

and $I_{i}: X \rightarrow X(i=1,2, \cdots)$ are appropriate functions.

As usual, we consider the corresponding Poincaré operator ([1]) defined by

$$
\mathbb{P}(\phi)=u_{T}(\phi),
$$

which maps an initial function $\phi$ along the unique mild solution $u(\phi)$ to problem (1.1)-(1.2) by $T$-units. We will present conditions and find methods to prove that these conditions ensure the existence of fixed points of the Poincaré operator. Clearly, such fixed point yields the $T$-periodic mild solutions to problem (1.1)-(1.2).

As noted in $[2,3,4,5,6,7,8,9,10]$, the study of problem (1.1)-(1.2) with infinite delay in general Banach spaces is difficult because the related Poincaré operator may not be compact. Therefore, fixed point theorems requiring compactness are not applicable to equations with infinite delay in general Banach spaces. Now, the combination of impulsive condition (1.3) makes problem (1.1)-(1.3) even more difficult.

These difficulties are overcome in [7] for $C_{g}$ (see [11] for more information on the space), where problem (1.1)-(1.3) are treated in a "weighted" Banach phase space $C_{g}$ defined by

$$
C_{g}=\left\{\phi: \phi \in P C((-\infty, 0], X) \text { and } \sup _{s \leq 0} \frac{\|\phi(s)\|}{g(s)}<+\infty\right\}
$$

which is equipped with the norm

$$
|\phi|_{g}=\sup _{s \leq 0} \frac{\|\phi(s)\|}{g(s)}, \quad \phi \in C_{g},
$$

where $P C((-\infty, 0], X)$ is the space of piecewise continuous functions from $(-\infty, 0]$ to $X, g$ is a function on $(-\infty, 0]$ such that

$$
g(0)=1, \quad g(-\infty)=+\infty,
$$

and $g$ is decreasing on $(-\infty, 0]$. Then the corresponding Poincaré operator is shown to be condensing with respect to the Kuratowski's measure of non-compactness in $C_{g}$. Hence, periodic solutions are derived by virtue of the Sadovskii's Fixed Point Theorem when solutions of problem (1.1)-(1.3) are locally strictly bounded.

In this paper, we will extend the results in $[5,6,7,9,10]$ to problem (1.1)-(1.3) in a "relaxed" fading memory phase space, which is defined to be a Banach space $(\mathscr{P},|\cdot| \mathscr{P})$ consisting of functions from $(-\infty, 0]$ to $X$ that satisfy the following axiom $([2,7,11,12])$ :

(A1). There exist a positive constant $H$ and locally bounded non-negative continuous functions $K(\cdot)$ and $M(\cdot)$ on $[0,+\infty)$ with the property that if $u:(-\infty, a) \rightarrow X$ is piecewise continuous on $[\sigma, a)$ with $u_{\sigma} \in \mathscr{P}$ for some $\sigma<a$, then for all $t \in[\sigma, a), u_{t} \in \mathscr{P}$, and

$$
H\|u(t)\| \leq\left|u_{t}\right|_{\mathscr{P}} \leq K(t-\sigma) \sup _{\sigma \leq s \leq t}\|u(s)\|+M(t-\sigma)\left|u_{\sigma}\right|_{\mathscr{P}} .
$$


A fading memory space is called a uniform fading memory space if it satisfies (A1) with $K(\cdot) \equiv K_{1}$ (a constant) and $M(t) \rightarrow 0$ as $t \rightarrow+\infty$.

We will first prove that the related Poincaré operator is condensing with respect to the Kuratowski's measure of non-compactness in general fading memory phase spaces.

Hence, periodic solutions are derived by using the Sadovskii's Fixed Point Theorem when solutions of problem (1.1)-(1.3) are locally strictly bounded. Also, if the phase spaces are uniform fading memory phase spaces, then by using a fixed point theorem of Hale and Lunel [13], we will make a classical extension by deriving periodic solutions when solutions of problem (1.1)-(1.3) are bounded and ultimate bounded. Since $C_{g}$ is a typical example of uniform fading memory phase spaces (cf. [11]), our results obtained in this paper extend those in $[5,6,7,9,10]$ (for equations without impulsive conditions or in special $C_{g}$ phase spaces) to problem (1.1)-(1.3) in these quite general fading memory phase spaces. In this way, we present a very general existence theorem of periodic solutions and related analysis method for problem (1.1)-(1.3) in general fading memory phase spaces.

\section{The Condenseness of the Poincaré Operator}

For problem (1.1)-(1.3), we make the following assumptions.

Assumption 2.1. For a constant $T>0, f(t+T, x, y)=f(t, x, y), A(t+T)=A(t), t \geq 0 . f$ is continuous in its variables and is locally Lipschitzian in the second and the third variables, and $f$ is bounded for all $(t, x, y), I_{i}, i=1,2, \cdots$, are Lipschitzian and compact. And

$$
0<t_{1}<t_{2}<\cdots<t_{p}<T<t_{p+1} \text { and } t_{p+k}=t_{k}+T, I_{p+k}=I_{k}, k \geq 1 .
$$

Assumption 2.2. [14, p.150] For $t \in[0, T]$ one has

(H1). The domain $D(A(t))=D$ is independent of $t$ and is dense in $X$.

(H2). For $t \geq 0$, the resolvent $R(\lambda, A(t))=(\lambda I-A(t))^{-1}$ exists for all $\lambda$ with $R e \lambda \leq 0$ and is compact, and there is a constant $M$ independent of $\lambda$ and $t$ such that

$$
\|R(\lambda, A(t))\| \leq M(|\lambda|+1)^{-1}, \operatorname{Re} \lambda \leq 0 .
$$

(H3). There exist constants $L>0$ and $0<a \leq 1$ such that

$$
\left\|(A(t)-A(s)) A(r)^{-1}\right\| \leq L|t-s|^{a}, s, t, r \in[0, T] .
$$

Assumption 2.3. For the fading memory space $\mathscr{P}$ satisfying (A1), we assume that $M(0)<1$.

Remark 2.4. In $C_{g}, M(0)=\frac{1}{2}$, see [9]. So, this assumption is satisfied there.

Under the Assumption 2.2, the results in, e.g., Amann [1] and Pazy [14] imply the existence of a unique evolution system $U(t, s), 0 \leq s \leq t \leq T$, for problem (1.1).

Let $\phi \in \mathscr{P}$ be fixed. We define a mild (in a generalized sense) solution (called solution throughout in this paper) of problem (1.1)-(1.3) to be a piecewise continuous function $u:(-\infty,+\infty) \rightarrow X$ with points of discontinuity $t_{i}$, where $u$ is left continuous and has the right limits, and satisfies $u(s)=\phi(s), s \leq 0$, and

$$
u(t)=U(t, 0) \phi(0)+\int_{0}^{t} U(t, h) f\left(h, u(h), u_{h}\right) d h+\sum_{0<t_{i}<t} U\left(t, t_{i}\right) I_{i}\left(u\left(t_{i}\right)\right), t \geq 0
$$


It is easily seen that mild solutions satisfy impulsive condition (1.3). Similar to $[5,6,7,9,10]$, to be able to study fixed points and periodic solutions, we assume that, for each $\phi \in \mathscr{P}$, problem (1.1)-(1.3) has a unique (mild) solution $u(\cdot, \phi)$ existing on $(-\infty,+\infty)$.

Now, we consider the Poincaré operator $\mathbb{P}: \mathscr{P} \rightarrow \mathscr{P}$ given by

$$
\begin{aligned}
\mathbb{P}(\phi) & =u_{T}(\cdot, \phi), \phi \in \mathscr{P}, \\
(\text { i.e., }(P \phi)(s) & \left.=u_{T}(s, \phi)=u(T+s, \phi), s \leq 0\right),
\end{aligned}
$$

which maps the initial function $\phi$ along the unique solution $u(\cdot, \phi)$ by $T$ units.

Definition 2.5. The Kuratowski's measure of non-compactness (or the $\alpha$ measure) for a bounded set $E$ of a Banach space $Y$ with norm $|\cdot|_{Y}$ is defined as

$$
\alpha(E)=\inf \{d>0: E \text { has a finite cover of diameter }<d\} .
$$

Definition 2.6. [13] Suppose that $\alpha$ is Kuratowski's measure of non-compactness in Banach space $Y$ and that $\mathbb{P}: Y \rightarrow Y$ is a continuous operator. Then $\mathbb{P}$ is said to be a condensing operator if $\mathbb{P}$ takes bounded sets into bounded sets, and $\alpha(\mathbb{P}(B))<\alpha(B)$ for every bounded set $B$ of $Y$ with $\alpha(B)>0$.

Let $\mathscr{D} \subset \mathscr{P}$ and $u(\phi)$ be the unique solution with $u_{0}(\phi)=\phi$. Then we write

$$
\begin{gathered}
\mathscr{P}_{l}(\mathscr{D})=\left\{u_{l}(\phi): \phi \in \mathscr{D}\right\}, \\
\mathscr{P}_{[h, r]}(\mathscr{D})=\left\{u_{[h, r]}(\phi): \phi \in \mathscr{D}\right\},
\end{gathered}
$$

where $u_{[h, r]}$ is the restriction of $u$ on $[h, r]$.

We have from [7] the following result.

Lemma 2.7. Let the Assumptions 2.1 and 2.2 be satisfied, and let $\mathscr{D} \subset \mathscr{P}$ be bounded. Then $\alpha(\mathscr{P}[l, r][(\mathscr{D}))=$ 0 for any $0<l<r \leq T$.

Now, combining the techniques from [5,7], we have the following result concerning the condenseness of the Poincaré operator $\mathbb{P}$.

Theorem 2.8. Let the Assumptions 2.1, 2.2, and 2.3 be satisfied. Then the operator $\mathbb{P}$ defined by (2.2) is condensing in $\mathscr{P}$.

Proof. First, we know from the proof of [7] and the Axiom (A1) that $\mathbb{P}$ is a continuous operator. It also implies that $\mathbb{P}$ takes bounded sets into bounded sets. Next, we can find from the assumption on function $M(\cdot)$ in the Assumption 2.3 an $\varepsilon \in(0,1)$ such that

$$
M(s) \leq \varepsilon, \quad s \in[0, \delta],
$$

for some $\delta>0$. Since $K(\cdot)$ and $M(\cdot)$ in the Axiom (A1) are locally bounded, there exist $\bar{K}$ and $\bar{M}$ such that

$$
K(t) \leq \bar{K}, \quad M(t) \leq \bar{M}, \quad t \in[0, T] .
$$

From our assumptions, we see that

$$
\begin{aligned}
U^{*} & :=\max _{0 \leq s \leq t \leq T}|U(t, s)|, \\
f^{*} & :=\max \{\|f(h, u, w)\|: \text { all }(h, u, w)\}
\end{aligned}
$$


are finite numbers. Let $0<\bar{r}<1$ and $\bar{\varepsilon}>0$ be constants. Then there exists an integer $N_{0}>1$ such that

$$
\left\{\begin{array}{l}
\varepsilon^{N_{0}-1}\left(\bar{K} \frac{U^{*}}{H}+\bar{M}\right)<\bar{r} \\
2 \varepsilon^{N_{0}-1} \bar{K} U^{*} f^{*} \frac{T}{N_{0}}<\bar{\varepsilon} \\
w_{0}:=\frac{T}{N_{0}}<\delta
\end{array}\right.
$$

From $[6,9]$, we know that

$$
\alpha\left(\mathscr{P}_{t}(\mathscr{D})\right) \leq K(t-\sigma) \alpha\left(\mathscr{P}_{[\sigma, t]}(\mathscr{D})\right)+M(t-\sigma) \alpha\left(\mathscr{P}_{\sigma}(\mathscr{D})\right), \quad 0 \leq \sigma \leq t .
$$

Hence it follows that from (2.7) and Lemma 2.7 that

$$
\begin{aligned}
\alpha(\mathbb{P}(\mathscr{D})) \leq & \alpha\left(\mathscr{P}_{T}(\mathscr{D})\right) \\
\leq & K\left(T-\left(T-w_{0}\right)\right) \alpha\left(\mathscr{P}_{\left[T-w_{0}, T\right]}(\mathscr{D})\right) \\
& +M\left(T-\left(T-w_{0}\right)\right) \alpha\left(\mathscr{P}_{T-w_{0}}(\mathscr{D})\right) \\
= & K\left(w_{0}\right) \alpha\left(\mathscr{P}_{\left[T-w_{0}, T\right]}(\mathscr{D})\right)+M\left(w_{0}\right) \alpha\left(\mathscr{P}_{T-w_{0}}(\mathscr{D})\right) \\
= & M\left(w_{0}\right) \alpha\left(\mathscr{P}_{T-w_{0}}(\mathscr{D})\right) \\
\leq & \varepsilon \alpha\left(\mathscr{P}_{T-w_{0}}(\mathscr{D})\right) \\
\leq & \varepsilon\left[K\left(w_{0}\right) \alpha\left(\mathscr{P}_{\left[T-2 w_{0}, T-w_{0}\right]}(\mathscr{D})\right)+M\left(w_{0}\right) \alpha\left(\mathscr{P}_{T-2 w_{0}}(\mathscr{D})\right)\right] \\
= & \varepsilon M\left(w_{0}\right) \alpha\left(\mathscr{P}_{T-2 w_{0}}(\mathscr{D})\right) \\
\leq & \varepsilon^{2} \alpha\left(\mathscr{P}_{T-2 w_{0}}(\mathscr{D})\right) \\
\cdots & \varepsilon^{N_{0}-1} \alpha\left(\mathscr{P}_{w_{0}}(\mathscr{D})\right) \\
\leq & \varepsilon^{N_{0}-1}\left[K\left(w_{0}\right) \alpha\left(\mathscr{P}_{\left[0, w_{0}\right]}(\mathscr{D})\right)+M\left(w_{0}\right) \alpha(\mathscr{D})\right] .
\end{aligned}
$$

Next, we see from the proof of [7] that

$$
\alpha\left(\mathscr{P}_{\left[0, w_{0}\right]}(\mathscr{D})\right) \leq \frac{U^{*}}{H} \alpha(\mathscr{D})+2 U^{*} f^{*} w_{0} .
$$

Using (2.6), (2.8), and (2.9), we obtain

$$
\begin{aligned}
\alpha(\mathbb{P}(\mathscr{D})) & \leq \varepsilon^{N_{0}-1}\left[K\left(w_{0}\right) \alpha\left(\mathscr{P}\left[0, w_{0}\right](\mathscr{D})\right)+M\left(w_{0}\right) \alpha(\mathscr{D})\right] \\
& \leq \varepsilon^{N_{0}-1}\left[\bar{K}\left(\frac{U^{*}}{H} \alpha(\mathscr{D})+2 U^{*} f^{*} w_{0}\right)+\bar{M} \alpha(\mathscr{D})\right] \\
& \leq \varepsilon^{N_{0}-1}\left[\left(\bar{K} \frac{U^{*}}{H}+\bar{M}\right) \alpha(\mathscr{D})+2 \bar{K} U^{*} f^{*} w_{0}\right] \\
& \leq \varepsilon^{N_{0}-1}\left(\bar{K} \frac{U^{*}}{H}+\bar{M}\right) \alpha(\mathscr{D})+2 \varepsilon^{N_{0}-1} \bar{K} U^{*} f^{*} \frac{T}{N_{0}} \\
& <\bar{r} \alpha(\mathscr{D})+\bar{\varepsilon} .
\end{aligned}
$$

Since $\bar{\varepsilon}>0$ is arbitrary, we get

$$
\alpha(\mathbb{P}(\mathscr{D})) \leq \bar{r} \alpha(\mathscr{D})<\alpha(\mathscr{D}) .
$$

This completes the proof. 


\section{EXISTENCE OF PERIODIC SOLUTIONS}

In this section, we present our existence theorem of periodic solutions to problem (1.1)-(1.3) by showing the existence of fixed points of the Poincare operator $\mathbb{P}$ defined by $(2.2)$, as it is verified in [7,9] that these fixed points give rise to periodic solutions. We first list some known fixed point theorems.

Theorem 3.1. (Sadovskii [15]) Let $\mathbb{P}$ be a condensing operator on a Banach space $Y$. If $\mathbb{P}(E) \subseteq E$ for a convex, closed and bounded set $E$ of $Y$, then $\mathbb{P}$ has a fixed point in $E$.

Theorem 3.2. (Hale and Lunel [13]) Suppose that $S_{0} \subseteq S_{1} \subseteq S_{2}$ are convex bounded subsets of a Banach space $Y, S_{0}$ and $S_{2}$ are closed, and $S_{1}$ is open in $S_{2}$. Suppose that $\mathbb{P}$ is a condensing operator in $Y$. If $\mathbb{P}^{j}\left(S_{1}\right) \subseteq S_{2}, j \geq 0$, and there is a number $N\left(S_{1}\right)$ such that $\mathbb{P}^{k}\left(S_{1}\right) \subseteq S_{0}, k \geq N\left(S_{1}\right)$, then $\mathbb{P}$ has a fixed point.

Note that the advantage of these fixed point theorems is that the compactness is not required so that we can try them on infinite delay impulsive evolution equations in general Banach spaces where the related Poincaré operator may not be a compact operator.

To apply Theorem 3.1, the solutions should be bounded locally; and to apply Theorem 3.2, the solutions should be bounded asymptotically. To make these more precise, we define the following.

Definition 3.3. The solutions of problem (1.1)-(1.3) are said to be locally strictly bounded if there exists a constant $C>0$ such that $|\phi|_{\mathscr{P}} \leq C$ implies that its solution satisfies $\|u(t, \phi)\| \leq C$ for $t \in[0, T]$.

Definition 3.4. The solutions of problem (1.1)-(1.3) are said to be bounded if, for each $B_{1}>0$, there is a $B_{2}>0$ such that $|\phi|_{\mathscr{P}} \leq B_{1}$ and $t \geq 0$ imply that its solution satisfies $\|u(t, \phi)\|<B_{2}$.

Definition 3.5. The solutions of problem (1.1)-(1.3) are said to be ultimate bounded if there is a bound $B>0$ such that, for each $B_{3}>0$, there is a $K>0$, such that $|\phi|_{\mathscr{P}} \leq B_{3}$ and $t \geq K$ imply that its solution satisfies $\|u(t, \phi)\|<B$.

Applying Theorem 3.1 with the notion of locally strictly boundedness, we obtain the following result.

Theorem 3.6. Let the Assumptions 2.1, 2.2, and 2.3 be satisfied. Assume that $K(T)+M(T) \leq 1$, where $K(\cdot)$ and $M(\cdot)$ are functions on $[0,+\infty)$ given in (A1). If the solutions of problem (1.1)-(1.3) are locally strictly bounded, then problem (1.1)-(1.3) has a T periodic solution.

Proof. Let the operator $\mathbb{P}$ be defined by (2.2). Write $Q:=\left\{\phi \in \mathscr{P}:|\phi|_{\mathscr{P}} \leq C\right\}$ with $C$ from Definition 3.3. Then $Q$ is convex, closed and bounded in $\mathscr{P}$. Next, for $u(\cdot)=u(\cdot, \phi)$ with $\phi \in Q$, the locally strictly boundedness implies that $\|u(t)\| \leq C$ for $t \in[0, T]$. Hence, for $\phi \in Q$, we have

$$
\begin{aligned}
|\mathbb{P}(\phi)|_{\mathscr{P}} & =\left|u_{T}(\phi)\right|_{\mathscr{P}} \\
& \leq K(T) \sup _{s \in[0, T]}\|u(s)\|+M(T)|\phi|_{\mathscr{P}} \\
& \leq[K(T)+M(T)] C \\
& \leq C .
\end{aligned}
$$

This means that

$$
\mathbb{P}(Q) \subset Q .
$$


Therefore, Theorem 3.1 tells us that there exists a fixed point for $\mathbb{P}$. It follows from [7, 9] that problem (1.1)-(1.3) has a $T$-periodic solution. This ends the proof of the theorem.

Applying Theorem 3.2 with the notion of bounded and ultimate boundedness, we obtain the following result.

Theorem 3.7. Let the Assumptions 2.1, 2.2, and 2.3 be satisfied, and let the phase space $\mathscr{P}$ be a uniform fading memory space. If the solutions of problem (1.1)-(1.3) are bounded and ultimate bounded, then problem (1.1)-(1.3) has a T-periodic solution.

Proof. Let the operator $\mathbb{P}$ be defined by (2.2). From [7, 9], we have

$$
\mathbb{P}^{m}(\phi)=u_{m T}(\phi), \phi \in \mathscr{P}, m=0,1,2, \cdots .
$$

Suppose that $B>0$ is the bound in the definition of ultimate boundedness. Then, the boundedness tells us that there is a $B_{1}>\left(K_{1}+1\right) B$ such that

$$
\left\{|\phi|_{\mathscr{P}} \leq\left(K_{1}+1\right) B, t \geq 0\right\} \text { implies }\|u(t, \phi)\|<B_{1},
$$

where $K_{1}$ is from the definition of a uniform fading memory space, and moreover, there is a $\bar{B}_{2}>B_{1}$ such that

$$
\left\{|\phi|_{\mathscr{P}} \leq B_{1}, t \geq 0\right\} \text { implies }\|u(t, \phi)\|<\bar{B}_{2} .
$$

Since the space is a uniform fading memory space, we see that there exists $\bar{M}$ such that $M(\cdot) \leq \bar{M}$. Let

$$
B_{2}>\max \left\{B_{1}, K_{1} \bar{B}_{2}+\bar{M} B_{1}\right\}
$$

and set

$$
\begin{aligned}
& S_{2}:=\left\{\phi \in \mathscr{P}:|\phi|_{\mathscr{P}} \leq B_{2}\right\}, \\
& S_{1}:=\left\{\phi \in \mathscr{P}:|\phi|_{\mathscr{P}}<B_{1}\right\}, \\
& S_{0}:=\left\{\phi \in \mathscr{P}:|\phi|_{\mathscr{P}} \leq\left(K_{1}+1\right) B\right\} .
\end{aligned}
$$

Then, we know that

$$
S_{0} \subseteq S_{1} \subseteq S_{2}
$$

are convex bounded subsets of $\mathscr{P}, S_{0}$ and $S_{2}$ are closed, and $S_{1}=S_{1} \cap S_{2}$ is open in $S_{2}$. Clearly, for $\phi \in S_{1}$ and $j \geq 0$, we have

$$
\begin{aligned}
\left|P^{j} \phi\right|_{\mathscr{P}} & =\left|u_{j T}(\phi)\right|_{\mathscr{P}} \\
& \leq K(j T) \sup _{0 \leq s \leq j T}\|u(s)\|+M(j T)\left|u_{0}\right|_{\mathscr{P}} \\
& \leq K(j T) \sup _{0 \leq s \leq j T}\|u(s)\|+M(j T)|\phi|_{\mathscr{P}} \\
& \leq K_{1} \bar{B}_{2}+\bar{M} B_{1} \\
& \leq B_{2},
\end{aligned}
$$

which implies that

$$
\mathbb{P}^{j}\left(S_{1}\right) \subseteq S_{2}, j \geq 0 .
$$

Next, we prove that there exists a number $N\left(S_{1}\right)$ such that

$$
\mathbb{P}^{k}\left(S_{1}\right) \subseteq S_{0} \text { for } k \geq N\left(S_{1}\right) .
$$


It follows from the ultimate boundedness that there exists a positive number $\sigma$ such that

$$
\left\{|\phi|_{\mathscr{P}} \leq B_{1}, t \geq \sigma\right\} \text { implies }\|u(t, \phi)\|<B
$$

Therefore, we have, for $\phi \in S_{1}$ and $k T>\sigma$,

$$
\begin{aligned}
\left|P^{k} \phi\right|_{\mathscr{P}} & =\left|u_{k T}(\phi)\right|_{\mathscr{P}} \\
& \leq K(k T-\sigma) \sup _{\sigma \leq s \leq k T}\|u(s)\|+M(k T-\sigma)\left|u_{\sigma}\right|_{\mathscr{P}} \\
& \leq K_{1} B+M(k T-\sigma)\left[K(\sigma) \sup _{0 \leq s \leq \sigma}\|u(s)\|+M(\sigma)|\phi|_{\mathscr{P}}\right] \\
& \leq K_{1} B+M(k T-\sigma)\left[K_{1} \bar{B}_{2}+\bar{M} B_{1}\right] \\
& \leq K_{1} B+M(k T-\sigma) B_{2} .
\end{aligned}
$$

Thus, as $M(t) \rightarrow 0(t \rightarrow+\infty)$ for the uniform fading memory space, we can find an integer $N\left(S_{1}\right)$ such that

$$
M(k T-\sigma) B_{2}<B \text { for } k \geq N\left(S_{1}\right)
$$

Hence, for $k \geq N\left(S_{1}\right)$, (3.5) becomes

$$
\begin{aligned}
\left|P^{k} \phi\right|_{\mathscr{P}} & \leq K_{1} B+M(k T-\sigma) B_{2} \\
& \leq\left(K_{1}+1\right) B
\end{aligned}
$$

which implies

$$
\mathbb{P}^{k}\left(S_{1}\right) \subseteq S_{0}, k \geq N\left(S_{1}\right)
$$

Consequently, Theorem 3.2 can be used to obtain a fixed point for operator $\mathbb{P}$, which, together with [7, 9], gives rise to a $T$-periodic solution of problem (1.1)-(1.3). This completes the proof.

Next, we remove the boundedness from Theorem 3.7.

Definition 3.8. The solutions of problem (1.1)-(1.3) are said to be locally bounded if for each $B_{1}>0$ and $K>0$, there is a $B_{2}>0$ such that $|\phi|_{\mathscr{P}} \leq B_{1}$ and $0 \leq t \leq K$ imply that its solution satisfies $\|u(t, \phi)\|<B_{2}$.

Theorem 3.9. ([10]) "Local boundedness and ultimate boundedness" implies "boundedness and ultimate boundedness".

Theorem 3.10. ([7]) Let the Assumptions 2.1, 2.2, and 2.3 be satisfied. Then the solutions of problem (1.1)-(1.3) are locally bounded.

Now, combining Theorems 3.7, 3.9, and 3.10, we have the following.

Theorem 3.11. Let the Assumptions 2.1, 2.2, and 2.3 be satisfied, and let the phase space $\mathscr{P}$ be a uniform fading memory space. If the solutions of problem (1.1)-(1.3) are ultimate bounded, then (1.1)-(1.3) has a T-periodic solution. 


\section{CONCLUSIONS AND SOME COMMENTS ON FUTURE STUDY}

By virtue of the Kuratowski measure of non-compactness, the properties of fading memory phase spaces and condensing operators, and the Sadovskii's fixed point theorem, it is shown that problem (1.1)(1.3), concerning impulsive differential equations with infinite delay in Banach spaces (1.1)-(1.3), admits a periodic solution under some basic assumptions, and this existence theorem for periodic solutions to problem (1.1)-(1.3) under the setting of the general fading memory phase spaces is a general extension of the results obtained in $[5,6,7,9,10]$ (for equations without impulsive conditions or in special $C_{g}$ phase spaces).

For the future study, it is meaningful to investigate the corresponding impulsive problem for fractional differential equations with infinite delay in Banach spaces. Clearly, for problem (1.1)-(1.3), the corresponding impulsive problem for fractional differential equations with infinite delay in Banach spaces could be as follows.

$$
\begin{aligned}
& { }^{c} D_{t}^{\alpha} u(t)+A(t) u(t)=f\left(t, u(t), u_{t}\right), t>0, t \neq t_{i}, \\
& u(s)=\phi(s), \quad s \leq 0, \\
& \Delta u\left(t_{i}\right)=I_{i}\left(u\left(t_{i}\right)\right), \quad i=1,2, \cdots, 0<t_{1}<t_{2}<\cdots<+\infty,
\end{aligned}
$$

where ${ }^{c} D_{t}^{\alpha}$ is the Caputo fractional differential operator of order $\alpha \in(0,1)$ (cf, e.g., $[16,17,18,19,20$, 21]).

Define

$$
\psi(t, s):=\alpha \int_{0}^{+\infty} \theta t^{\alpha-1} \xi_{\alpha}(\theta) \exp \left(-t^{\alpha} \theta A(s)\right) d \theta,
$$

where $\xi_{\alpha}$ is a probability density function on $(0,+\infty)$ such that its Laplace transform is given by

$$
\begin{aligned}
& \int_{0}^{+\infty} e^{-\sigma x} \xi_{\alpha}(\sigma) d \sigma=\sum_{j=0}^{+\infty} \frac{(-x)^{j}}{\Gamma(1+\alpha j)}, 0<\alpha \leq 1, x>0 . \\
& \varphi_{1}(t, \tau):=[A(t)-A(\tau)] \psi(t-\tau, \tau), \\
& \varphi_{k+1}(t, \tau):=\int_{\tau}^{t} \varphi_{k}(t, s) \varphi_{1}(s, \tau) d s, k=1,2, \cdots, \\
& \varphi(t, \tau):=\sum_{k=1}^{+\infty} \varphi_{k}(t, \tau), \\
& U(t):=-A(t) A^{-1}(0)-\int_{0}^{t} \varphi(t, s) A(s) A^{-1}(0) d s .
\end{aligned}
$$

As showed in [20], under some conditions, one could try to give a definition of mild solutions to problem (4.1)-(4.3) with the help of these families of operators above. Thus, it is quite possible to obtain similar results for problem (4.1)-(4.3) as what we have done above for problem (1.1)-(1.3). We would like to remark here that one should be very careful for dealing with fractional differential problem (4.1)(4.3) since the impulsive condition and infinite delay could make things complex. Furthermore, it is also meaningful to investigate the existence of asymptotically periodic solutions and S-asymptotically $\omega$-periodic solutions to the corresponding impulsive problem for fractional differential equations based on [17].

\section{Funding}


The first author was supported by the National Natural Science Foundation of China under Grant No. 11571229. The fourth author was supported by the National Natural Science Foundation of China under Grant No. 11771091 and the Fudan University under Grant No. IDH 1411016.

\section{REFERENCES}

[1] H. Amann, Periodic solutions of semilinear parabolic equations, In: Cesari, L., Kannan, R., Weinberger, R. (eds.) Nonlinear Analysis, A Collection of Papers in Honor of Erich H. Rothe, pp. 1-29. Academic Press, New York (1978).

[2] T.A. Burton, B. Zhang, Periodic solutions of abstract differential equations with infinite delay, J. Differential Equations 90 (1991), 357-396.

[3] D.N. Chalishajar, Controllability of second order impulsive neutral functional differential inclusions with infinite delay, J. Optim. Theory Appl. 154 (2012), 672-684.

[4] D.N. Chalishajar, K. Karthikeyan, A. Anguraj, Existence results for impulsive perturbed partial neutral functional differential equations in Frechet spaces, Dyn. Contin. Discrete Impuls. Syst. Ser. A Math. Anal. 22 (2015), 25-45.

[5] K. Ezzinbi, J. Liu, N. Minh, Periodic solutions in fading memory spaces, Discrete and Continuous Dynamical Systems, Supplement Vol. (2005), 250-257.

[6] H. Henriquez, Periodic solutions of quasi-linear partial functional differential equations with unbounded delay, Funkcial. Ekvac. 37 (1994), 329-343.

[7] J. Liang, J.H. Liu, T.J. Xiao, Condensing operators and periodic solutions of infinite delay impulsive evolution equations, Discrete Contin. Dyn. Syst. Ser. S 10 (2017), 475-485.

[8] J. Liang, J.H. Liu, T.J. Xiao, H.K. Xu, Periodicity of solutions to the Cauchy problem for nonautonomous impulsive delay evolution equations in Banach spaces, Anal. Appl. 15 (2017), 57-475.

[9] J.H. Liu, Periodic solutions of infinite delay evolution equations, J. Math. Anal. Appl. 247 (2000), 627-644.

[10] J.H. Liu, T. Naito, N. Minh, Bounded and Periodic solutions of infinite delay evolution equations, J. Math. Anal. Appl. 286 (2003), 705-712.

[11] B. Zhang, Space $C_{g}$ and uniform behavior of solutions of functional differential equations with infinite delay, Acta Math. Sinica (N.S.) 4 (1988), 177-188.

[12] J. Liang, T.J. Xiao, Solvability of the Cauchy problem for infinite delay equations, Nonlinear Anal. 58 (2004), $271-297$.

[13] J. Hale, S. Lunel, Introduction to Functional Differential Equations, Springer-Verlag, New York, 1993.

[14] A. Pazy, Semigroups of linear operators and applications to partial differential equations, Springer-Verlag, New York, 1983.

[15] B. Sadovskii, On a fixed point principle, (Russian) Funkcional. Anal. i Priložen. 1 (1967), 74-76.

[16] G.M. Mophou, G. N'Guérékata, Existence of mild solution for some fractional differential equations with nonlocal conditions, Semigroup Forum 79 (2009), 315-322.

[17] T.A. Burton, B. Zhang, Asymptotically periodic solutions of fractional differential equations, Dyn. Contin. Discrete Impuls. Syst. Ser. A Math. Anal. 20 (2013), 1-21.

[18] D.N. Chalishajar, K. Karthikeyan, Existence and uniqueness results for boundary value problems of higher order fractional integro-differential equations involving Gronwall's inequality in Banach spaces, Acta Math. Sci. Ser. B Engl. Ed. 33 (2013), 758-772.

[19] T. Diagana, G.M. Mophou, G.M. N'Guérékata, On the existence of mild solutions to some semilinear fractional integrodifferential equations, Elect. J. Quali. Theory Diff. Equ. 58 (2010), 1-17.

[20] M. M. El-Borai, The fundamental solutions for fractional evolution equations of parabolic type, J. Appl. Math. Stoch. Anal. 3 (2004), 197-211.

[21] F. Li, Existence for nonautonomous fractional integrodifferential equations with nonlocal conditions, Abstr. Appl. Anal. 2013 (2013), Article ID 723453. 